\title{
Hypertension in Cushing's Syndrome: From Pathogenesis to Treatment
}

\author{
Maria Verena Cicala Franco Mantero \\ Division of Endocrinology, University of Padua, Padua, Italy
}

\section{Key Words}

Cushing's syndrome $\cdot$ Resistant hypertension $\cdot$ Multiple therapy

\begin{abstract}
Hypertension is one of the most distinguishing features of endogenous Cushing's syndrome (CS), as it is present in about $80 \%$ of adult patients whereas in children its prevalence is about $47 \%$. Hypertension in CS is significantly correlated with the duration of hypercortisolism and results from the interplay between several pathophysiological mechanisms regulating plasma volume, peripheral vascular resistance and cardiac output, all of which are increased in this state. Glucocorticoids cause hypertension through several mechanisms: their intrinsic mineralocorticoid activity; through activation of the renin-angiotensin system; by enhancement of vasoactive substances, and by causing suppression of the vasodilatory systems. In addition, glucocorticoids may exert some hypertensive effects on cardiovascular regulation through the CNS via both glucocorticoid and mineralocorticoid receptors. Hypertension in CS usually resolves with surgical removal of the tumor, but some patients require pharmacological antihypertensive treatment both pre- and postoperatively. Thiazides and furosemide should be avoided, while adrenergic blockade and calcium channel antagonists are usually ineffective. Mineralocorticoid receptor antagonists, Ang II blockers and ACE inhibitors are good
\end{abstract}

anti-hypertensive options; PPAR- $\gamma$ agonists may help in many aspects of the insulin resistance syndrome. The relatively selective glucocorticoid receptor antagonist Mifepristone (RU 486) could reduce blood pressure in patients with CS. Neuromodulatory agents such as the serotonin inhibitors cyproheptadine and ritanserin, valproid acid, dopamine agonists, somatostatin analogs may occasionally be effective, as well as drugs acting directly at the adrenal levels, such as Ketoconazole and aminoglutetimide or even opDDD. Treating hypertension in CS remains a difficult task and a big challenge, in order to decrease the morbidity and mortality associated with the disease.

Copyright $\odot 2010$ S. Karger AG, Basel

\section{Introduction}

A chronic excess of glucocorticoid exposure can cause a rare disorder called Cushing's syndrome (CS). Adrenal causes include autonomous cortisol production by a unilateral benign or malignant adenoma, or bilateral adrenal hyperplasia, or dysplasia. Nonadrenal causes result from adrenocorticotropin (ACTH) overproduction, due to a corticotropic adenoma (Cushing's disease); an extrapituitary (ectopic) ACTH-producing tumor or, rarely, an ectopic corticotropin-releasing hormone-producing tumor, leading to excess cortisol production. Additionally, exogenous steroids can cause iatrogenic CS.

\section{KARGER}

Fax +4161306 1234 E-Mail karger@karger.ch www.karger.com
(C) 2010 S. Karger AG, Basel

0028-3835/10/0925-0044\$26.00/0

Accessible online at:

www.karger.com/nen
Franco Mantero

Department of Medical and Surgical Sciences

Division of Endocrinology, University Clinic Padua

Via Ospedale 105, IT-35128 Padua (Italy)

Tel. +39 049821 3000, Fax +39 049657 391, E-Mail franco.mantero@ unipd.it 
The overall incidence of endogenous CS is approximately 2-5 new cases per million population per year. In most cases, the onset of the disease is insidious and it usually takes months to years for the full-blown symptomatology to develop [1].

The prevalence of hypertension in adult patients with endogenous CS is approximately $80 \%$ (but it rises to $95 \%$ in patients with ectopic ACTH secretion), whereas in children and adolescents it is about $47 \%$ [1]. The majority of patients with CS present with mild-to-moderate hypertension, whereas $17 \%$ could present with a severe form. The reported prevalence may be related to the degree or the cause of hypercortisolism and is significantly correlated with the duration of hypercortisolemia. Cosecretion of other steroids may also influence the degree and the response to treatment. The hypertension of endogenous CS is characterized by disruption of the blood pressure (BP) circadian rhythm, with loss of the physiological nocturnal fall.

In iatrogenic CS, the prevalence of hypertension is about $20 \%$ and it is dose-dependent.

In patients with ectopic ACTH syndrome over 95\% present with hypertension. This form of CS is characterized by signs of mineralocorticoid excess, with hypokalemic alkalosis found in $95-100 \%$ of cases, in contrast to less than $10 \%$ in other forms of CS.

\section{Pathogenesis of Hypertension in CS}

Hypertension in CS results from an interplay of several pathophysiological mechanisms (table 1) regulating plasma volume, peripheral vascular resistance, and cardiac output, all of which are increased in CS. In addition, glucocorticoids may exert some hypertensive effects on cardiovascular regulation through the central nervous system via both glucocorticoid and mineralocorticoid receptors (MR). Ultimately, glucocorticoids induce changes that increase cardiac output, total peripheral resistance and renovascular resistance [2], leading to chronic hypertension. Insulin resistance and sleep apnea seem also to contribute to the hypertension of CS.

\section{Mineralocorticoid Activity of Cortisol}

The mechanism of glucocorticoid-induced hypertension has been largely elucidated with the discovery of the enzyme $11 \beta$-hydroxysteroid dehydrogenase (11ß-HSD) type 2.

Cortisol binds to both glucocorticoid and MR. The MR is held to be nonselective. However, the plasma levels
Table 1. Mechanisms of hypertension and of resistant hypertension in CS

- Mineralocorticoid activity of cortisol

- Activation of the renin-angiotensin system

- Enhancement of cardiovascular reactivity to vasoconstrictors (catecholamines, vasopressin and angiotensin II

- Increased $\beta$-adrenergic receptor sensitivity to catecholamines

- Suppression of the vasodilatory systems (NO synthase, prostacyclin and kinin-kallikrein)

- Increased cardiac output, total peripheral resistance and renovascular resistance

- Insulin resistance, sleep apnea

of cortisol are 100- to 1,000-fold higher than those of aldosterone, which suggests that the MR could be predominantly activated by the glucocorticoid. Instead, the MR is protected from cortisol binding by the $11 \beta-\mathrm{HSD} 2$, a gate-keeping enzyme, which is critical for modulating corticosteroid hormone action at tissue level. Actually, two forms of $11 \beta$-HSD have been isolated: the type 1 isoform, 11 $\beta$-HSD1 (a NADP $[\mathrm{H}]$-dependent enzyme) catalyses both dehydrogenation and reduction and it is held to interconvert cortisol to cortisone. $11 \beta-\mathrm{HSD} 1$ is most abundantly expressed in the liver and adipose tissue. The type 2 isoform $11 \beta$-HSD2 (NAD-dependent) is active at very low cortisol concentrations and has mainly dehydrogenase activity, thus inactivating cortisol to cortisone. It is highly expressed in mineralocorticoid target tissues, such as renal cortex, colon, salivary and sweat glands.

Both $11 \beta$-HSD type 1 and 2 are present in vascular endothelial cells, coronary artery cells, and vascular smooth cells, suggesting that they function to modulate local access of cortisol to the vasculature.

Cortisol in a physiological concentration range is prevented from binding to MR in the mineralocorticoid target tissues by the locally produced $11 \beta-H S D 2$. However, excess cortisol levels like those in CS might exceed the capacity of $11 \beta-H S D 2$ to inactivate the steroid to cortisone, thus making it available to bind to the MR and mimic excess aldosterone (fig. 1) [3]. The ensuing MR activation results in increased renal tubular sodium reabsorption and intravascular volume expansion.

At a cellular and molecular level mineralocorticoid hypertension derives from an overactivity of the epithelial $\mathrm{Na}^{+}$channel $(\mathrm{ENaC})$, the gradient-driven sodium channel located in the apical membrane of the cells in the distal tubule and cortical collecting duct of the kidney. The resulting blood volume expansion suppresses endogenous plasma renin activity. Since $\mathrm{Na}^{+}$re-absorption in 
Fig. 1. Peripheral cortisol metabolism and MR selectivity. Cortisol and aldosterone bind with equal affinity to the MR. Plasma concentrations of cortisol are much higher than those of aldosterone, but in mineralocorticoid-responsive cells the 11ß-HSD2 enzyme converts cortisol to the receptorinactive form cortisone, permitting aldosterone to occupy the receptor. The hemiacetal conformation of the 11-hydroxyl group with the 18-aldehyde group of aldosterone renders this steroid a poor substrate for the enzyme.

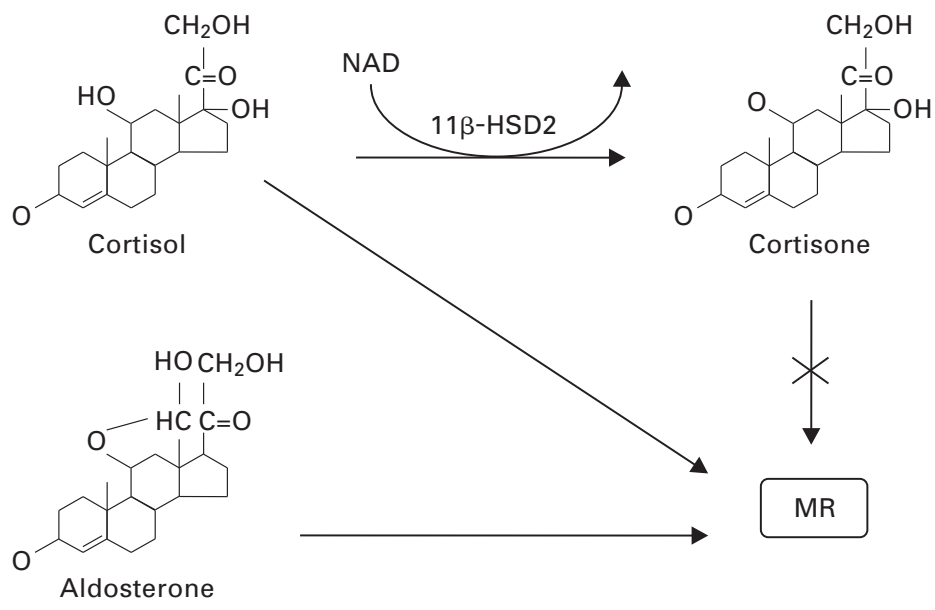

with the degree of hypokalemia. In contrast with AME syndrome, the THE values are normal in CS, and they are even slightly higher in patients with the highest ectopic ACTH production. Therefore, it is the markedly elevated serum cortisol levels that are responsible for the high THF + allo THF/THE ratio as opposed to a low THE. These data lead to the speculation that substrate saturation and spillover of cortisol to the MR receptor can explain the functional mineralocorticoid excess state seen in these hypokalemic patients. However, a direct action of ACTH on the activity of $11 \beta-H S D 2$ has also been suggested [6].

\section{Activation of the Renin-Angiotensin System}

Recently, it was demonstrated that glucocorticoids increase angiotensinogen. Despite this, plasma renin activity and plasma renin concentration are generally normal or suppressed in patients with CS, suggesting that there is an overall increased throughput in the system [8]. Moreover, patients with CS show an increased pressor response to angiotensin II, suggesting increased sensitivity to the agent. Glucocorticoids also enhance central actions of angiotensin II to increase BP [9].

\section{Enhancement of Cardiovascular Reactivity to \\ Vasoconstrictors}

Endothelin-1 (ET-1), the most potent vasoconstrictor peptide with marked hypertensive, mitogenic and atherogenic effects, is significantly elevated in patients with 
untreated active CS. A trend towards reduction in plasma ET was observed in the patients after successful treatment and normalization of cortisol levels.

The catabolic effect of hypercortisolism promotes endothelial damage and increases vascular permeability, which in turn leads to ET-1 hypersecretion in the circulation and thus exerts a permissive effect on the local atherogenic and pressor action of ET-1 leading to endothelial dysfunction that is typically associated with arterial hypertension and CS. Therefore, the raised plasma ET-1 levels can be considered a biochemical marker of endothelial dysfunction.

Moreover, cortisol-induced hypertension is characterized by a reduced activity of the nitric oxide (NO) depressor system, which leads, by itself, to an increase of ET [10].

Glucocorticoids also appear to downregulate the expression of the $\mathrm{Na}^{+}, \mathrm{Ca}^{2+}$ exchanger in vascular smooth muscle cells, which elevates cytosolic $\mathrm{Ca}^{2+}$ and causes vasoconstriction. Moreover, cortisol increases the serum concentration of erythropoietin and causes polycythemia, a well-recognized complication of chronic glucocorticoid excess. Erythropoietin has a direct vasoconstrictor effect and might be a further mediator of cortisol-induced hypertension.

\section{Increased $\beta$-Adrenergic Receptor Sensitivity to}

\section{Catecholamines}

In a state of glucocorticoid excess the sympathetic nervous system is upregulated through the augmentation of the action of catecholamines. Catecholamine levels and receptors are normal, but $\beta$-adrenergic receptor sensitivity to catecholamines is increased, with a consequently increased vascular tone.

\section{Suppression of the Vasodilatory Systems}

NO, a well-known vasodilator in humans, is produced via the enzyme NO synthase (NOS). Glucocorticoids inhibit NOS synthesis and may lead to increased BP by reduction in peripheral vasodilation [11].

Similarly, glucocorticoids inhibit the production of prostacyclin, another potent vasodilator in the vascular endothelium. Other vasodilators, PGE2 and kallikrein, are decreased in CS patients [7].

\section{Insulin Resistance and Sleep Apnea}

Although the main mechanism of hypertension and resistant hypertension in CS is overstimulation of the nonselective MR by cortisol, other causes, such as insulin resistance and sleep apnea, are major contributors to hy- pertension in CS patients. Insulin resistance is commonly associated with hypertension as a part of the metabolic syndrome, leading to increased morbidity and mortality [12]. In patients with CS, insulin resistance results from chronic glucocorticoid exposure and leads to sodium and water retention and volume expansion. Moreover, excess of glucocorticoids, and consequently hyperinsulinemia, causes increased sympathoadrenal system activity, local renin-angiotensin aldosterone system activation, and vascular hypertrophy, leading to increased vascular resistance and hypertension [12].

Sleep apnea occurs also as a cause of hypertension in CS. Disproportionate weight gain in the head, neck, and waist can partially explain obstructive sleep apnea in CS. Sleep apnea can worsen hypertension in CS through increased sympathetic tone during hypoxemic episodes, associated insulin resistance and diabetic autonomic neuropathy [13].

\section{Treatment of Glucocorticoid-Induced Hypertension}

Hypertension in CS usually resolves with surgical removal of the tumor, but some patients require pharmacological antihypertensive treatment, both pre- and postoperatively, to decrease $\mathrm{BP}$ and the duration of hypertension.

The hypertension of CS is significantly correlated with the duration of hypercortisolism. In most cases, months to years often pass before the diagnosis is suspected and ultimately confirmed, particularly in patients with milder expressions of the disorder. The therapeutic goal is to find and remove the cause of excess glucocorticoids. Therapeutic strategies for CS-specific hypertension (to normalize BP and decrease the duration of hypertension) are necessary to decrease morbidity and mortality associated with this disease.

\section{Surgical Treatment}

The treatment of choice depends upon the cause of the hypercortisolism, which must be established unequivocally. Optimal treatment is the correction of hypercortisolism without permanent dependence on hormone replacement. Patients with ectopic tumors and primary adrenal disease are treated with surgical tumor excision and bilateral or unilateral (in the case of unilateral adrenal adenomas or carcinomas) adrenalectomy, respectively. For Cushing's disease, selective transsphenoidal resection of ACTH-secreting pituitary adenomas is the initial treatment of choice. After 'curative' surgery, systolic and 
Table 2. Therapeutic options in the medical treatment of hypertension in Cushing's disease

- Spironolactone (effective in mineralocorticoid excess)

- Eplerenone (selective aldosterone antagonist)

- Sartans

- Angiotensin-converting enzyme inhibitors

- Inhibitors of steroidogenesis

- Selective glucocorticoid receptor antagonism with mifepristone (RU 486)

- Serotonin inhibitors (cyproheptadine, ritanserin)

- Bromocriptine, octreotide, valproic acid (lower ACTH concentrations)

- Peroxisome proliferator-activated receptor $\gamma$-agonists (rosiglitasone, pioglitasone)

diastolic BP fall, but roughly one-third of adult patients continue to have systolic and three-quarters have diastolic hypertension [1]. No strong correlation was found between the severity of hypertension before cure and the persistence of hypertension after cure. There was, however, a positive correlation between the duration of hypertension preoperatively and the persistence of hypertension after surgery. This probably reflects irreversible remodeling of the vasculature due to long-standing hypertension [14].

In children and adolescents with CS, hypertension usually improves one year after surgery, suggesting that young patients with hypercortisolism are unlikely to have developed irreversible vascular remodeling. Despite a significant improvement after surgical cure, children with CS are at risk for residual hypertension, as demonstrated by the group of Stratakis [15]. This suggests that pediatric patients with Cushing's disease be followed closely for cardiovascular morbidity associated with hypertension.

\section{Pharmacological Therapy}

A significant proportion of patients do not achieve complete cure after surgical treatment or require a prolonged period of time for complete response to therapy. In such cases, pharmacological antihypertensive treatment should be used, both pre- and post-operatively. Understanding the pathophysiology of hypertension in CS is crucial for making the decision regarding the pharmacological agents to be used in the treatment of these patients (table 2) [16].

In general, more than a single agent is required to reach the recommended target $\mathrm{BP}$ levels. Thiazides and furosemide can worsen the hypokalemia and therefore should be avoided, unless adrenergic blockade and calcium channel antagonists are usually less effective. Because glucocorticoid hypertension may be augmented by the renin-angiotensin system, sartans and ACE inhibitors are recommended. Until recently, spironolactone and his metabolites canrenone and potassium canrenoate were the only MR antagonists available, usually effective in the cases where mineralocorticoid excess is obvious. Eplerenone, a novel selective aldosterone antagonist with limited adverse effects, may be beneficial in the treatment of hypertension in CS, but it requires higher doses and repeated administration [1]. The sartans also lower BP in CS patients, as do the ACE inhibitors, while no data are available for the direct renin inhibitor aliskiren. Blockade of the renin-angiotensin system with sartans or ACE inhibitors in hypertensive CS patients results in amelioration of hypertension and normalization of BP in approximately $50 \%$ of the cases [17]. In general, hypertension can be difficult to treat or be resistant to treatment in the setting of CS without direct relief from the hypercortisolism.

Several drugs that suppress steroidogenesis, such as ketoconazole, aminoglutethimide, metyrapone or opDDD, may ameliorate hypertension [16].

Peroxisome proliferator-activated receptor- $\gamma$ agonists such as rosiglitasone or pioglitasone, have been tried in patients with Cushing's disease. These agents have a positive impact on many aspects of the insulin resistance syndrome, including hypertension and microalbuminuria, and could reduce cardiovascular risk factors in Cushing's disease patients. However, their role in ACTH and cortisol reduction is not so effective and unwanted effects such as weight gain have been reported. As some sartans also have peroxisome proliferator-activated receptor- $\gamma$ agonist activity they should probably be preferred in CS [18].

The relatively selective glucocorticoid receptor antagonist mifepristone (RU 486) could reduce BP in patients with CS. Neuromodulatory agents such as the serotonin inhibitors cyproheptadine and ritanserin, valproic acid, dopamine agonists, somatostatin analogs may occasionally be effective [16].

A new multi-receptor targeted somatostatin analogue (Pasireotide) is currently under evaluation in the treatment of Cushing's disease with promising preliminary results in the inhibition of urinary free cortisol. 


\section{Conclusions}

Treating hypertension in CS remains a difficult task and a big challenge, in order to decrease the morbidity and mortality associated with the disease. In fact, the combination of hypertension with several comorbidities (central obesity, impaired glucose tolerance, dyslipidemia, coagulative disorders) is responsible for increased cardiovascular complications (coronary artery disease, heart failure and cardiac infarction); this high cardiovascular risk leads to a mortality rate from time higher than expected [19].

Eighty percent of patients with Cushing's disease present with hypertension resulting from an interplay of several pathophysiological mechanisms. In addition to CSspecific hypertension, patients with CS, with a developmental or genetic predisposition, often develop essential hypertension as well. The hypertension is more likely to become resistant in this setting.

The control of hypersecretion of cortisol by surgical, neurosurgical, or pharmacologic means, as well as the blockade of glucocorticoid and MR are effective means of lowering BP in most hypertensive Cushing's disease patients and of normalizing it in approximately 50\%. The patients who do not achieve normotension or may require a prolonged period of time for the correction of hypercortisolism usually need concomitant or sequential use of multiple antihypertensive treatment modalities to reduce $\mathrm{BP}$ and the duration of hypertension.

\section{Disclosure Statement}

The authors have nothing to disclose.

\section{References}

1 Magiakou MA, Smyrnaki P, Chrousos GP: Hypertension in Cushing's syndrome. Best Pract Res Clin Endocrinol Metab 2006;20: 467-482.

2 Muiesan ML, Lupia M, Salvetti M, Grigoletto C, Sonino N, Boscaro M, Rosei EA, Mantero F, Fallo F: Left ventricular structural and functional characteristics in Cushing's syndrome. J Am Coll Cardiol 2003;41:22752279.

3 Felix J, Frey A, Odermatt B, Frey M: Glucocorticoid-mediated mineralocorticoid receptor activation and hypertension. Curr Opin Nephrol Hypertens 2004;13:451-458.

4 Ferrari P, Bonny O: Forms of mineralocorticoid hypertension. Vitam Horm 2003;66: 113-156.

5 Ulick S, Chan CK, Gill JR Jr, Gutkin M, Letcher L, Mantero F, New MI: Defective fasciculata zone function as the mechanism of glucocorticoid-remediable aldosteronism. J Clin Endocrinal Metab 1990;71:1151-1157.

6 6 Quinkler M, Stewart PM: Hypertension and the cortisol-cortisone shuttle. J Clin Endocrinol Metab 2003;88:2384-2392.
7 Ferrari P, Krozowski Z: Role of the 1lbetahydroxysteroid dehydrogenase type 2 in blood pressure regulation. Kidney Int 2000; 57:1374-1381

8 Baid S, Nieman LK: Glucocorticoid excess and hypertension. Curr Hypertens Rep 2004;6:493-499.

9 Scheuer DA, Becht AG: Glucocorticoids potentiate central actions of angiotensin to increase arterial pressure. Am J Physiol Regul Integr Comp Physiol 2001;280:1719-1726. 10 Kirilov G, Tomova A, Dakovska L, Kumanov
P, Shinkov A, Alexandrov AS: Elevated plasma endothelin as an additional cardiovascular risk factor in patients with Cushing's syndrome. Eur J Endocrinol 2003;149:549-553.

11 Mangos GJ, Whitworth JA, Williamson PM: Glucocorticoids and the kidney. Nephrology 2003;8:267-273.

12 McFarlane SI, Banerji M, Sowers JR: Insulin resistance and cardiovascular disease. J Clin Endocrinol Metab 2001;86:713-718.

13 Sacerdote A, Weiss K, Tran T, Rokeya Noor B, McFarlane SI: Hypertension in patients with Cushing's disease: pathophysiology, diagnosis, and management. Curr Hypertens Rep 2005;7:212-218.
14 Bernini G, Moretti A, Iacconi A, et al: Anthropometric, hemodynamic, humoral, and hormonal evaluation in patients with incidental adrenocortical adenomas before and after surgery. Eur J Endocrinol 2003;148: 213-219.

15 Lodish MB, Sinaii N, Patronas N, Batista DL, Keil M, Samuel J, Moran J, Verma S, Popovic J, Stratakis CA: Blood pressure in pediatric patients with Cushing syndrome. J Clin Endocrinol Metab 2009;94:2002-2008.

16 Morris D, Grossman A: The medical management of Cushing's syndrome. Ann NY Acad Sci 2002;970:119-133.

17 El-Atat FA, Nicasio J, Clark LT, et al: An overview of the beneficial cardiovascular effects of thiazolidinediones. Therapy $2005 ; 2$ : $113 \mathrm{e}-119 \mathrm{e}$

18 Rossi GP: Losartan metabolite EXP3179: an AT1-receptor-independent treatment strategy for patients with the metabolic syndrome? Hypertension 2009;54:710-712.

19 Mancini T, Kola B, Mantero F, Boscaro M, Arnaldi G: High cardiovascular risk in patients with Cushing's syndrome according to 1999 WHO/ISH guidelines. Clin Endocrinol 2004;61:768-777. 